\title{
“Universal" curve of ionic conductivities in binary alkali borate glasses
}

\author{
MARCIO LUIS FERREIRA NASCIMENTO*, SHIGUEO WATANABE \\ Institute of Physics, University of São Paulo, Rua do Matão 187. São Paulo 05508-900, Brazil \\ E-mail: pmlfn@iris.ufscar.br
}

Since the discovery of fast ionic conductivity in glasses, there has been a large interest in explaining the diffusion mechanism. With this knowledge one may be able to design new glasses with optimized properties for various applications. It is well known that the ionic conductivity increases rapidly when a network glassformer is modified by the addition of a metal alkali. Despite considerable experimental and theoretical effort, there is currently no consensus regarding the diffusion mechanism [1]. Several models have been proposed: they vary from thermodynamics with principles in models for liquid electrolytes, such as the weak electrolyte model [2], to models based on solid state concepts such as the jump diffusion model [3], the strong electrolyte model [4], and the dynamic structure model [5].

Ionic conductivity $\sigma$ in glass is a thermally activated process of mobile ions by surmounting a potential barrier $E_{\mathrm{A}}$, of the form:

$$
\sigma=\sigma_{0} \exp \left(-E_{\mathrm{A}} / k_{\mathrm{B}} T\right)
$$

where $\sigma_{0}$ is the pre-exponential factor, $k_{\mathrm{B}}$ is the Boltzmann constant and $T$ is the temperature. Extensive studies have recently been made for obtaining an "universal" equation from the standpoint of glass structure. For example, Doi [6] presented conductivity values of 17 different glasses, not mentioned, that follows an "universal" conductivity rule. Swenson and Börjesson [7] proposed a common cubic scaling relation of $\sigma$ with the expansion volumes of the networking forming units in salt-doped and-undoped glasses. This fact suggested that the glass network expansion, which is related to the available free volume, is a key parameter determining the increase of the high ionic conductivity in some types of fast ion conducting glasses.

According to Adams and Swenson [8], the ion conduction should be determined by the ionic motion within an infinite pathway cluster. For various silver ion conducting glasses $[9,10]$, it was found that the cubic root of the volume fraction $F$ of infinite pathways for a fixed valence mismatch threshold is closely related to both the absolute conductivity and the activation energy of the conduction process:

$$
\log _{10} \sigma T \propto \sqrt[3]{F} \approx E_{\mathrm{A}} / k_{\mathrm{B}} T
$$

Fig. 1 shows arrhenian plots of $\sigma$ for 49 binary alkali borate glasses, of form $x \mathrm{~A}_{2} \mathrm{O} \cdot(1-x) \mathrm{B}_{2} \mathrm{O}_{3}(\mathrm{~A}=$
$\mathrm{Li}, \mathrm{Na}, \mathrm{K}, \mathrm{Cs}, x$ in mol\%, indicated [11]), ranging from $10^{-1} \mathrm{~S} / \mathrm{cm}$ to less than $10^{-20} \mathrm{~S} / \mathrm{cm}$ between $20^{\circ} \mathrm{C}$ and $400^{\circ} \mathrm{C}$. The range of activation energy $E_{\mathrm{A}}$ lie between 0.4 and $1.5 \mathrm{eV}$ in all glasses studied. These data were compared with "universal" equation for $\sigma_{0}=50 \mathrm{~S} / \mathrm{cm}$ in Equation 1. The "universal" equation, following Doy's sense, appears in Fig. 1 as a dotted line.

The substitution of the mobile ion by another kind of mobile ion introduces modification of the glass structure, as well as its ionic conductivity in various ways. Therefore, the results shown in Fig. 1 is remarkable in the sense that so many different binary alkali borate glasses present curves of $\log _{10} \sigma$ vs. $E_{\mathrm{A}} / k_{\mathrm{B}} T$ very close to each other and to the "universal curve". There is a strong correlation between then $\sigma$ and $E_{\mathrm{A}}$ values in a wide range of temperatures. It is interesting to note that the increase in ionic conductivity with alkali content is almost entirely due to the fact that the activation energy $E_{\mathrm{A}}$ required for a cation jump decreases, as presented in Ref. [4].

Thus, the $\sigma_{0}$-value in Equation 1 is practically unaffected by alkali content. Actually, $\sigma_{0}=50 \mathrm{~S} / \mathrm{cm}$ is an average value of all the $\sigma_{0}$-values of the glasses considered in Fig. 1. Therefore, maybe, instead of an "universal" curve, one should use an "average" curve.

It is important to note that the $\sigma$-values for several binary alkali borate glasses lie close to an "universal" curve. Although the $\sigma$-values for each glass at very low and very high temperatures differ by more than 19 orders of magnitude, for a given value of $E_{\mathrm{A}} / k_{\mathrm{B}} T$, the difference between large to small values of $\sigma$ is only one order of magnitude in $90 \%$ of the glass systems considered in Fig. 1. Cesium borate glasses were the most prominent exception. This means that, once obtained $\sigma$-value at a given temperature, the approximate value of $E_{\mathrm{A}}$ can be obtained from the "universal" equation, i.e., taking $\sigma_{0}=50 \mathrm{~S} / \mathrm{cm}$. The values of $\sigma$ for different temperatures can then be obtained using this $E_{\mathrm{A}}$-value. Of course, if $E_{\mathrm{A}}$-value is obtained by some other way, the ionic conductivity of a glass can be calculated using Equation 1.

Another "universal" curve, following Equation 2, was obtained, and is presented in Fig. 2. The preexponential value was $\sigma_{0}^{\prime}=50000 \mathrm{~K} \mathrm{~S} / \mathrm{cm}$, considering the same conductivity data of Fig. 1. The conclusions for this case also follows the above described considering Equation 1. 


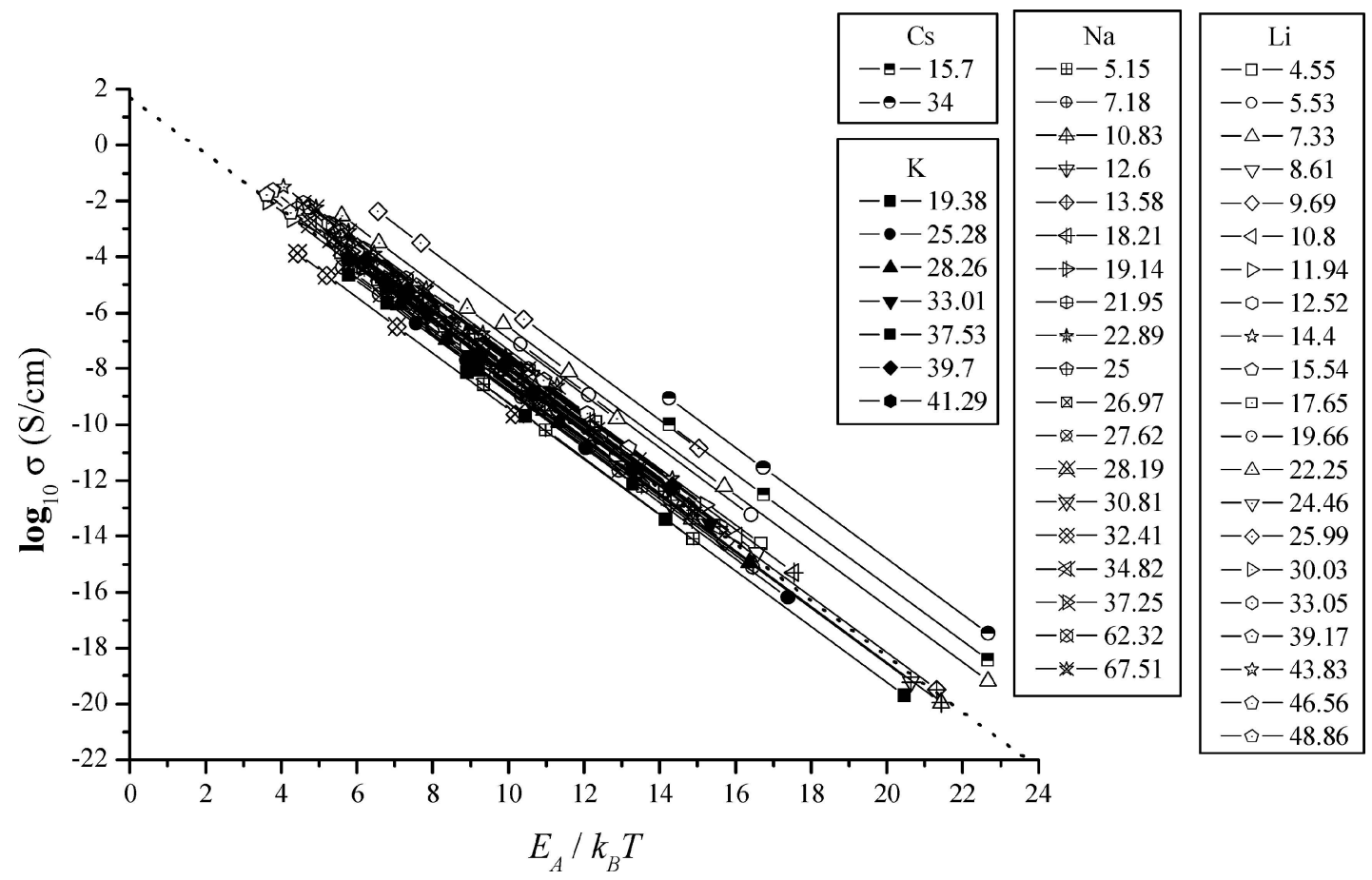

Figure 1 Arrhenian plots of ionic conductivities in 49 binary alkali borate glasses [11], of form $x \mathrm{~A}_{2} \mathrm{O} \cdot(1-x) \mathrm{B}_{2} \mathrm{O}_{3}(\mathrm{~A}=\mathrm{Li}, \mathrm{Na}, \mathrm{K}, \mathrm{Cs}, x$ in mol\%, indicated). The dotted line is an "universal curve", Equation 1, with $\sigma_{0}=50 \mathrm{~S} / \mathrm{cm}$.

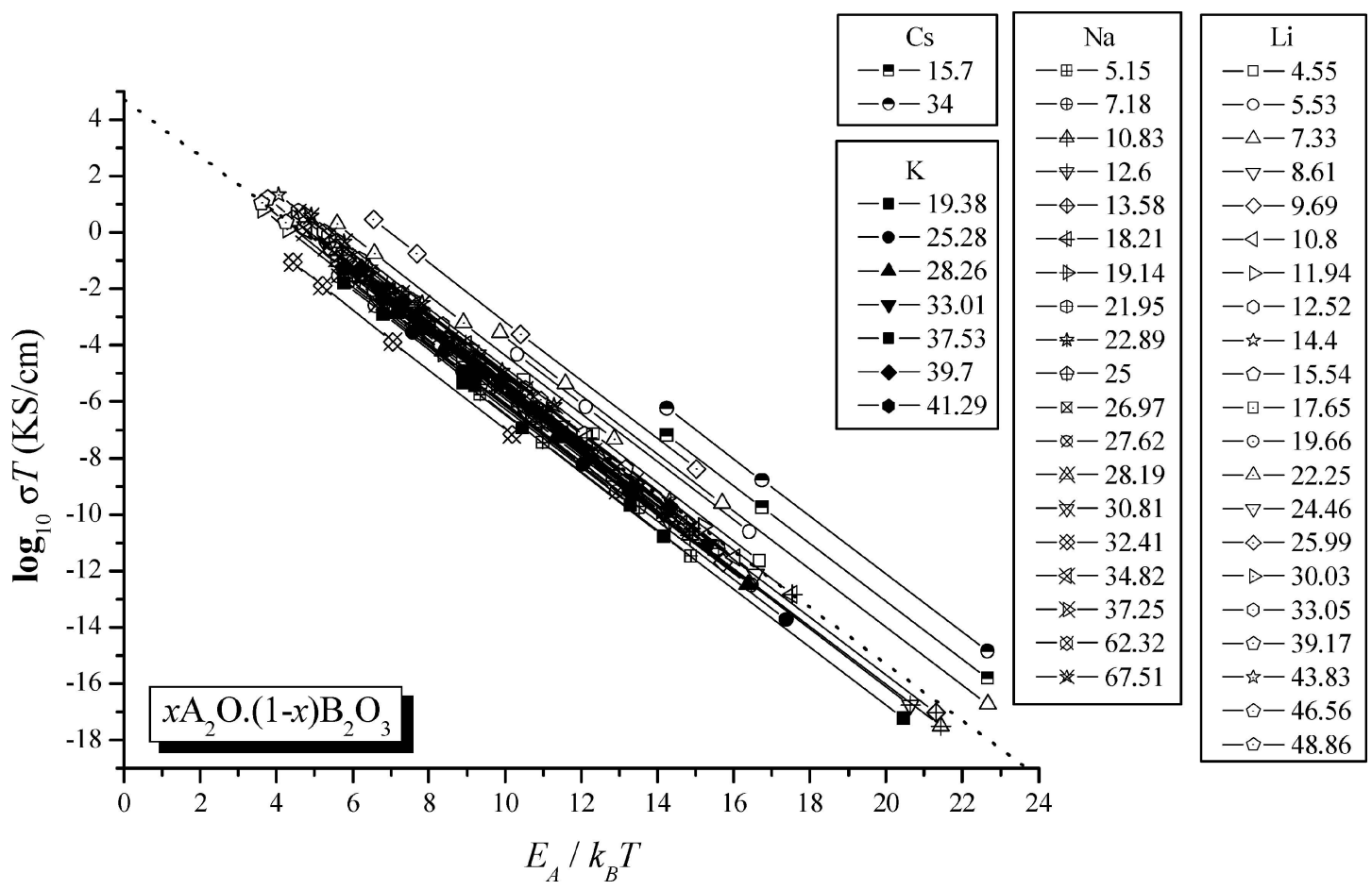

Figure 2 Arrhenian plots of ionic conductivities in 49 binary alkali borate glasses [11], of form $x \mathrm{~A}_{2} \mathrm{O} \cdot(1-x) \mathrm{B}_{2} \mathrm{O}_{3}(\mathrm{~A}=\mathrm{Li}, \mathrm{Na}, \mathrm{K}, \mathrm{Cs}, x$ in mol\%, indicated). The dotted line is an 'universal curve', Equation 2, with $\sigma_{0}^{\prime}=50000 \mathrm{~K} \mathrm{~S} / \mathrm{cm}$.

The reason for the decrease of the activation energy with increasing alkali concentration is, however, not clear. The expansion of the glass skeleton and the introduction of the alkali ions in voids in the structure forming narrow pathways would lead to two effects that lower the activation energy and thus promote the ionic conductivity. In terms of the Anderson-Stuart model [4], $E_{\mathrm{A}}$ may be written as a sum of two terms, which are denoted by the binding energy, $E_{\mathrm{b}}$, and the strain energy, $E_{\mathrm{s}}$. The binding energy is the average energy that a cation requires to leave its site, and $E_{\mathrm{s}}$ is the average ki- netic energy that a cation needs to structurally distort the environment and to create a "doorway" through which it can diffuse to a new site. The cation induced expansion of the network skeleton would lead to a lowering of the strain energy part $E_{\mathrm{s}}$ of the activation energy and the formation of pathways, in which the cations may coordinate with oxygens of the network, leading to a lowering of $E_{\mathrm{b}}$.

Further studies on other binary oxide glassformers as presented on Figs 1 and 2 will refuse or recognize this "universal" finding. 


\section{References}

1. C. A. ANGell, Ann. Rev. Phys. Chem. 43 (1992) 693.

2. D. RAvaine and J. L. Souquet, Phys. Chem. Glass. 18 (1977) 27.

3. K. FUn Ke, Prog. Solid State Chem. 22 (1993) 11.

4. O. L. ANDERSON and D. A. STUART, J.Am. Ceram. Soc. 37 (1954) 573.

5. A. BUNDE, M. D. INGRAM and P. MAASS, J. Non-Cryst. Solids 172/174 (1994) 1222.

6. A. DOI, J. Mater. Sci. 39 (2004) 6827.

7. J. SWENSON and L. B Ö RJESS ON, Phys. Rev. Lett. 77 (1996) 3569.
8. S. ADAMS and J. SWenson, Phys. Chem. Chem. Phys. 4 (2002) 3179.

9. J. SWENSON and S. ADAMS, Phys. Rev. B 64 (2002) 024204 .

10. S. AD A MS and J. SWEN S ON, Solid State Ion. 154/155 (2002) 151.

11. Sciglass ${ }^{\circledR}$ Database. Available at http://www.esm-software.com/ sciglass

Received 11 November 2004 and accepted 18 January 2005 